\title{
Multi-perspective Process Variability: A Case for Smart Green Buildings (Short Paper)
}

\author{
Aitor Murguzur \\ Information Technologies Area \\ IK4-Ikerlan Research Center, Spain \\ e-mail: amurguzur@ikerlan.es
}

\author{
Hong-Linh Truong and Schahram Dustdar \\ Distributed Systems Group \\ Vienna University of Technology, Austria \\ e-mail: \{truong,dustdar\}@dsg.tuwien.ac.at
}

\begin{abstract}
The variability scale in large-scale Cyber-Physical Systems (CPSs) is high and complex due to the voluminousness, dynamicity and diversity of available computing resources (people, things and software services), domain-specific processes, domain-specific elements (stakeholders, assets and contracts), and their relationships. This requires us to go beyond current variability modeling and management techniques which neglect the complexity and the diversity of relevant stakeholders, data and assets, and thus cannot cope with intelligent business and analytics requirements in dynamic environments, such as smart city management. In this paper, we present a comprehensive analysis for understanding the multi-perspective variability in processes atop people, data and things in CPSs, particularly, for the sustainability governance of Smart Green Buildings (SGBs). We examine domain-specific processes and domainspecific elements and their relationships to derive a multipleperspective variability management for SGBs. On the basis of this, we conceptualize a novel model for the multi-perspective process variability representation.
\end{abstract}

Keywords-smart city; smart building; variability; process configuration;

\section{INTRODUCTION}

Recently, the integration and high availability of people, data and things have introduced several platforms for sustainability analysis and governance of large-scale CyberPhysical Systems (CPSs), such as Smart Green Building (SGB) and smart city management [1]. Generally, sustainability analysis and governance in those settings deal with the maintenance and optimization of Mechanical, Electrical and Plumbing (MEP) systems and buildings to meet stakeholders' business objectives, e.g. reducing the energy consumption, and to comply with sustainability regulations, e.g. reducing Greenhouse Gas (GHG) emissions [2].

From our work of cloud-based M2M (Machine-toMachine) platforms for large-scale CPSs [3], we have observed that while people, data and things may be abstracted under the service model and invoked via different process models, existing techniques have not been adequate for supporting a large number of processes, which are entirely or partially common to several stakeholders and different assets to which domain processes are applied, to manage various complex types of variability. This is partially due to the emerging sustainability analysis and governance for largescale CPSs, but also because there is a lack of understanding of the relationship among stakeholders, processes, data and assets in smart environments. To tackle these challenges, we contribute (i) a comprehensive multi-perspective variability analysis of stakeholders and operation processes in SGBs, and (ii) a conceptual model representation of multiperspective process variability for SGBs.

The rest of the paper is organized as follows: Section II presents a multi-perspective variability analysis. Section III details our approach to model multi-perspective process variability. Related work is discussed in Section IV. Finally, we conclude the paper in Section V.

\section{Multi-Perspective Process Variability ANALYSIS IN SGBS}

Basically, smart buildings look at two major concerns [4]: (i) improving user experience and (ii) maintaining building sustainability. However, these two factors are normally competing in a sense of conflict of interests, and SGB solutions thus need to be sensitive to both within all sustainability governance life-cycle phases and related stakeholders.

The sustainability governance life-cycle in an SGB consists of four main phases. For the surveying phase, a number of services are needed for control and inspection tasks (e.g. online re-configuration of monitored equipment parameters) to ensure the correct and sustainable building operation. For the installation and commissioning phase, services (e.g. equipment procurement) are tailored and offered in accordance with each client's requirements. For the configuration phase, services include basic guiding principles that are used as guidelines in selecting an adequate building configuration depending on building types, regulations and stakeholders' requirements. In the operation phase, services need to be customized for several stakeholders involved in different tasks (e.g. monitoring of building facilities), which require combining sets of data, artifacts, applications and processes.

On the other hand, the variability may be observed from the domain perspective. A set of domain-specific processes (i.e. installation and commissioning, configuration, operation and surveying processes) are defined to manage, control 


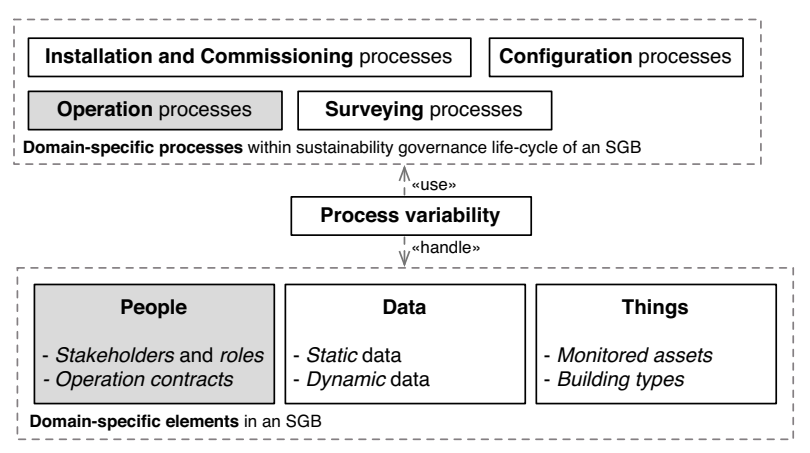

Figure 1. Domain processes and domain variability in an SGB ecosystem.

and monitor stakeholders - representing related roles and actors, operation contracts - which encompass different agreement policies among stakeholders, monitoring data which collects data from different assets, monitored assets including building facilities and monitored equipment, and building types, as illustrated in Fig. 1. Thus, such processes may be related to diverse domain-specific elements.

With respect to people, stakeholders, roles and associated operation contracts will need complex process models that access and utilize different applications, sub-processes, software and human services, and data types, which give rise to a large collection of related process variants (i.e. process family). These process variants essentially share a common part of a core process, whereas concrete parts fluctuate from variant to variant [5]. Concerning data, process models variability might be modeled and managed considering types of monitoring data - static monitoring data which is known before instantiation (e.g. data about building equipment), and dynamic monitoring data which is collected just-intime and cannot be anticipated (e.g. GHG data). In terms of things, beyond the people and data viewpoint, process variants could also be created for the same purpose (e.g. energy efficiency, energy consumption) depending on the diversity of monitored assets (e.g. building facilities - floor, room, etc., and equipment - chiller, boiler, etc.) and building types (e.g. residential, commercial).

Such domain-specific processes may have common parts that could be used to satisfy different domain-specific elements. For instance, given solutions for a particular task carried out by several stakeholders (e.g. Fault Detection and Diagnosis (FDD) of chillers in an SGB [6]), a process model can be built from common parts and manifold fragments due to monitored assets (i.e. types of chillers) and monitoring data (e.g. energy consumption of chillers). Therefore, from the position of a sustainability governance platform offering cloud-based service models for multiple stakeholders in a particular domain (e.g. SGBs, smart logistics, smart healthcare), we must deal with process variability identifying commonalities and variability in such process variants in order to deal with the complexity and heterogeneity.

\section{A. Stakeholders}

To understand the complexity and degree of the variability of domain-specific processes among stakeholders' diversity in SGBs, we need to analyze and perform a systematic classification of stakeholders and their common interesting assets. We classify stakeholders into six main groups, as shown in Fig. 2 with their respective interactions:

- Designers are involved in the construction of sustainable SGBs, equipped with intelligent control systems, efficient Heating, Ventilation and Air Conditioning (HVAC) systems, lighting, renewable solutions, energy storage systems and insulation that work together to achieve lower energy consumption and greater comfort.

- Owners are interested in the energy consumption of SGBs and the related billing of tenants. The energy information could go into more analysis about the performance or efficiency of individual assets (e.g. floors). Individuals and Organizations are grouped here.

- Providers offer the basis for enabling SGBs. Business service providers are divided into three sub-classes:

- Equipment providers are building technology Original Equipment Manufacturers (OEMs), which offer hardware that can be integrated into an SGB.

- Platform providers supply the governance platform that is integrated into SGBs' physical infrastructure. Various types of execution platforms exist to enhance complex functionalities and support on stakeholders' engagement and demand side on SGB governance.

- Application providers empower techniques for data monitoring, analysis and prediction.

- Operators are commissioned to manage an SGB and offer services based on provided monitored assets and monitoring data. They are divided into sub-classes:

- Building operators compare and verify energy consumption and enable user comfort considering tenants' requirements. For instance, data gathering will assist in FDD, target policy re-configuration and device adjustment.

- Auditors examine claimed data, techniques and applications, and certify them for their compliance with regulations (e.g. governments).

- Third-party operators have a contract with the owner, tenant or provider to perform activities on behalf of them (e.g. check energy consumption).

- Tenants require information about several dimensions of an SGB, so data should be prepared for different plans (e.g. requested thermal user comfort). Tenants (i.e. occupants) of certain parts of an SGB may provide data about their own spaces, equipment, etc.

- Community group access datasets and processes to offer services for the community and related stakeholders, exploiting data from different sources. 


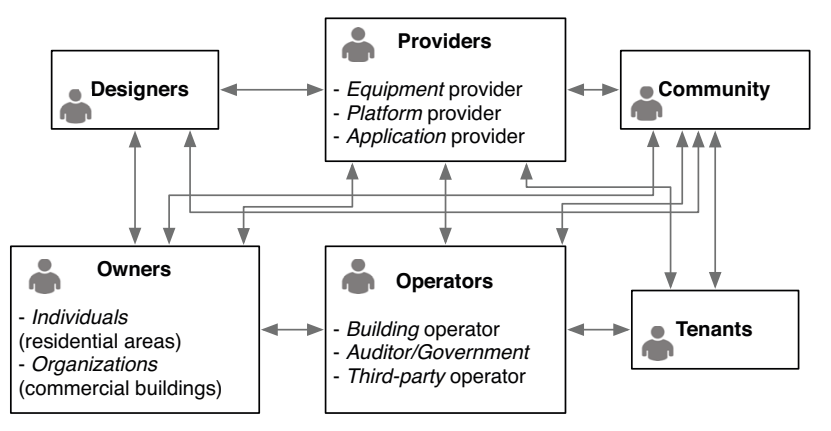

Figure 2. Main stakeholders and their possible interactions in an SGB ecosystem ( $\rightarrow$ representing interactions).

\section{B. Operation Processes in Sustainability Governance}

The operation phase encompasses all stakeholders and roles, consequently process identification and management in this phase becomes crucial to get an overview and understand relationships among stakeholders, monitoring data and respective monitored assets. From the analysis of various requirement documents, data and real-world services in M2M platforms for smart cities ${ }^{1}$ [7], [6], [8], [9], we have extracted some of the main categories of operation processes (OPs) for understanding process models variability in SGBs:

1) Building health status maintenance processes check the building deterioration level considering data from sensors to determine if design parameters, materials, structure, etc. cross a failure threshold level. This helps in tuning some aspects of the operational building and safety, as well as detecting correlations and good practices for future SGB solutions.

2) Individual equipment maintenance processes verify the status of installed equipment and systems in order to reduce errors in the anomaly-based detection of monitoring data through the combined analysis. If errors are detected, the maintenance process could assist providers (OEMs) and operators in decision making (e.g. re-configuring pre-established parameters) [7].

3) Electricity system maintenance processes provide inspection, maintenance and repair activities for all sizes of nonrenewable (e.g. fossil-fuel power stations) and renewable electricity systems (e.g. solar systems). Tasks are related to inspection of damage, inspection of electrical cable connections, checking of switches and circuit breakers or solar panel cleaning.

4) Mechanical system maintenance processes monitor and check mechanical infrastructure (e.g. elevators) against anomalies in order to empower mechanical (maintenance) engineers to predict costs, detect errors, improve performance, make better informed design decisions and explore more design options.

\footnotetext{
${ }^{1}$ Apart from the cited sources, we analyzed the Galaxy platform (http: //pacific-galaxy.com). However, due to confidential conditions, we could not publish documents, data, and processes.
}

5) Platform maintenance processes gather platform specific data to improve the virtual appliance performance (e.g. response time), change pre-determined parameters (e.g. scalability) and error detection to resolve critical tenant issues (e.g. server down). Application related activities are supported here.

6) Energy consumption processes are independent with energy types and energy consuming elements, which aim to examine energy consuming equipment and production equipment in near real-time. They also provide visualization of the total GHG emission amount of the entire building allowing tenants to lower their electricity bill and reduce energy by adjusting monitored devices (e.g. adjusting chiller configuration [6]).

7) Tenant billing processes deal with particular tenant consumed energy billing activities by eliminating manual meter readings for simplified, more accurate and automated measurement and payment verification.

8) Energy efficiency processes pursue energy saving through visualization of power usage quantities of different monitored assets [8]. In such processes, the power usage amount can be automatically collected in a pre-defined time frame (e.g. every $15 \mathrm{~min}$ ). The collected data is displayed on the power indicators together with the target values for energy saving of the building (e.g. lobby, room). They may also directly act on monitored assets (e.g. via actuators).

9) Demand monitoring and prediction processes offer demand monitoring through consumption prediction and "peak cut" of incoming power. Contracted electricity is monitored through process activities in order to control power consumption at peak hours. Also, these processes may have activities for single demand as well as multiple demands so that demand controls of multiple SGB buildings are managed, gathering the predicted power consumption amounts of each device, and comparing them to the threshold values [9].

10) Data analysis processes collect specific data from monitored assets. Therefore, facilities' deterioration level could be detected and predicted to invoke any condition-based maintenance process if necessary [6].

11) Compliance with regulations processes examine monitoring data from distinct assets and certify their compliance with regulations. These processes contain activities connected with humans, in which related stakeholders are notified when irregularities are found.

12) User comfort monitoring processes monitor and adjust automatically monitored elements based on predefined tenants' comfort policies (e.g. analyzing indoor air quality ${ }^{2}$ ) and monitoring data, giving users sustainable and valuable recommendations.

\footnotetext{
${ }^{2}$ The United States Environmental Protection Agency indoor air quality models, methods and databases: http://www.epa.gov/nrmrl/appcd/mmd.html
} 
The list of the above-mentioned OPs is by no means exhaustive. Still, it shows that for OPs in large-scale CPSs, different levels of variability exist, e.g. an OP may have process variants for different stakeholders and assets to which the process will be applied (e.g. the energy consumption process can be considered by all stakeholders except building solution designers and platform providers).

\section{Conceptual Model of Multi-Perspective PROCESS VARIABILITY IN SGBS}

Regarding the domain-specific elements of an SGB (see Fig. 3), the subset of stakeholders from above-mentioned ones and their respective roles, members of a building type (e.g. commercial or residential buildings) interact in different ways with the provided cloud-based system and its monitored assets. The latter encompass a number of building facilities (e.g. building, floor, department or room) as well as equipment (e.g. meters, lighting, and HVAC), with which different stakeholders and roles are able to manage, control and monitor them depending on a particular operation contract (e.g. premium or basic) and monitoring data values (e.g. building temperature and energy consumption of a chiller).

Domain-specific processes not only include previously introduced domain OPs but also embrace a set of installation and commissioning processes, configuration processes and surveying processes. Those processes should be common for all SGB solutions abstracting commonalities and particularities for all stakeholders, roles, and operations contracts. Therefore, multi-perspective process variability is a combination of domain variability and process variability for a particular domain (in this case for SGBs). In other words, multi-perspective process variability represents the set of all possible perspectives of people (e.g. stakeholders, roles and operation contracts) in a particular domain, which includes different process variants to deal with the diversity and complexity of software services from supported applications, monitoring data (e.g. static and dynamic) and things (e.g. building types and monitored assets).

Therefore, process variability of a particular SGB can be managed at different levels (see Fig. 3):

- Single-perspective process variability: Given a single installation and commissioning process and focusing on a single stakeholder only, we might manage, control and monitor different types of equipment (e.g. chiller, boiler, HVAC, IT equipment), which may include common and variable functionalities (e.g. two types of chillers may have identical activity regarding status checking). The same could be applied to other levels (e.g. building facilities).

- Multi-perspective process variability: Given a single installation and commissioning process and a number of stakeholders, roles and operation contracts, other perspectives are inherent to a single-perspective process variability case. Apart from dealing with things variability, this process should include activities related to people variability in order to support multiple dimensions and create a specific view for each stakeholder.

In our view, a perspective describes a pre-configuration of a reference process model, i.e. it considers the aggregation of those stakeholders' views related to an operation contract (i.e. a viewpoint) under consideration. To handle such multiperspective process variability, a unique configurable process model to represent common parts of process variants, and a variability model to gather features and relationships among those stakeholders, roles, operation contracts, monitoring data, monitored assets and building types, and concrete parts representing variants (i.e. process fragments) are required.

Bearing in mind the conceptual model, we demonstrate an example to illustrate the practical feasibility of the presented model by modeling multi-perspective process variability of an elevators remote predictive maintenance and monitoring process in a SGB domain using two separated variability models - one for people perspective and the other one for things perspective ${ }^{3}$. The presented conceptual model has been implemented atop our LateVa toolkit [10].

\section{RELATED WORK}

Previous attempts, in form of surveys, have been realized to analyze the stakeholders from sustainability perspective, e.g. from sustainable cloud-based systems [4], [3] viewpoint. However, we provide a systematic classification of stakeholders in order to understand domain processes that they could be directly participating in the design and execution of SGB solutions and thus manage complex associations. The same goes for operation processes, i.e. althought some attempts [7], [8], [9] have been made to describe analytics processses (i.e. energy effiency of a building [8]), none of them list and provide a description of operation processes.

Regarding the process variability, several proposals have appeared in the literature over the last decade [5], [11], [12]. The PESOA project [5] was carried out to propose an approach for the development and customization of process-oriented software families. VxBPEL [11] enables capture of variation points, variants, and realization relations between variation points within a BPEL process extending ActiveBPEL for variability support in servicecentric systems. Baresi et al. [12] present an fragment-based re-use approach based on CVL and BPEL for managing process re-configuration at runtime. The latter approach uses DyBPEL which enables the adaptation of BPEL processes. These all proposals may be suitable for representing process variability. However, they have been focused on providing a variability management solution to handle process variants from a single perspective.

Just a few studies have been focused on multi-perspective process variability modeling and management [13], [14].

${ }^{3}$ Detailed information about the examples is available at: http:// aitormurguzur.com/projects/lateva/multi-perspective 


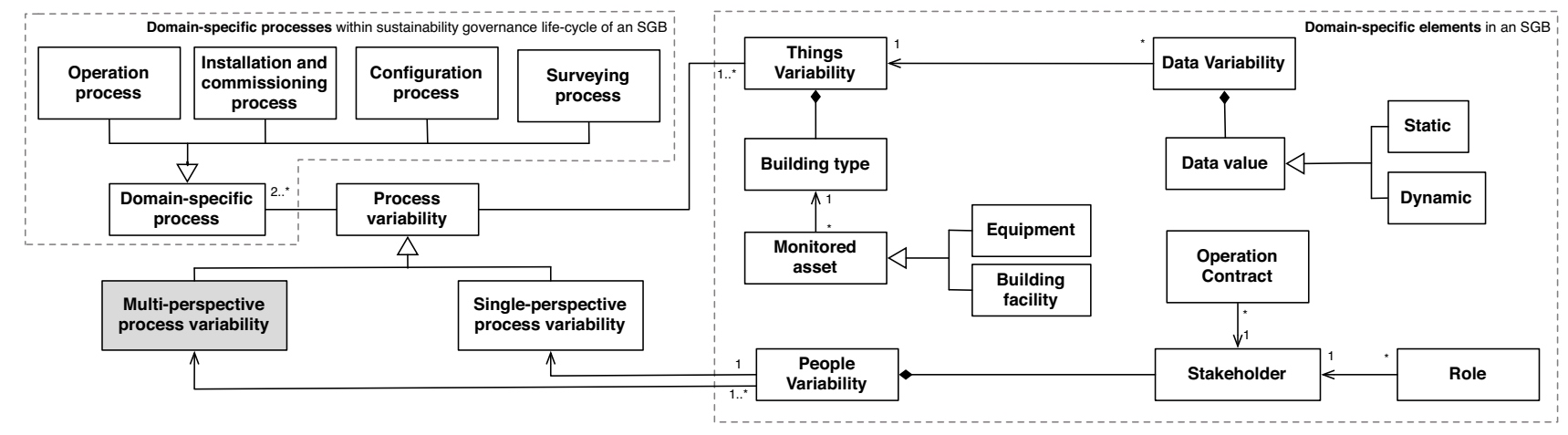

Figure 3. A conceptual model of multi-perspective process variability in SGB solutions.

The C-iEPC [13] is presented as an extension of the C-EPC notation with the notions of roles and objects, augmenting control-flow perspective. In parallel, the multi-perspective variants approach [14] differentiates between different levels of abstraction, involving different types of domain experts in the configuration process and finally reducing the number of decisions the user has to make during modeling and/or execution. With respect to the mentioned work, the main differences of our approach are two-fold: we have not focused on process elements variability modeling itself (i.e. how to deal with control-flow, data and resources variability in a process), instead we use multi-perspective process variability to deal with the complexity and diversity of large-scale CPSs and domain-specific elements at a lower level. Secondly, those two approaches use a single configurable process model to model multi-perspective variability, while our approach clearly separates domain-specific processes and domain-specific elements variability representation by using a fragment based re-use approach, separating commonality and variability representation. This improves scalability and offers more flexibility.

\section{CONClusions And Future Work}

Process variability may no longer only be about people and software integration, but needs to interact and utilize the multiplicity of sensors, devices and data that exist in dynamic environments. Understanding and modeling multiperspective process variability could bring benefit to stakeholders working in large-scale CPSs (as exemplified by SGB analysis), who are dealing with a large number of process collections and variants. From our detailed analysis of stakeholders and operation processes in SGBs, we introduced a multi-perspective process variability conceptual model capable of capturing people, data and things variability. This model has been integrated in our toolkit. First experiences are encouraging but we need much more tests to verify the effectiveness of our approach. In the near future, we also plan to allow easy and effective changes of multi-perspective models at runtime. Finally, to scale up our approach, we aim at evaluating it in terms of functionalities as well as in terms of performance, using more real world SGB scenarios.

\section{ACKNOWLEDGMENT}

This work is partially funded by the Pacific Controls Cloud Computing Lab (PC3L).

\section{REFERENCES}

[1] L. Sha, S. Gopalakrishnan, X. Liu, and Q. Wang, "CyberPhysical Systems: A New Frontier," in SUTC, 2008.

[2] L. Gurgen, O. Gunalp, Y. Benazzouz, and M. Gallissot, "Self-aware cyber-physical systems and applications in smart buildings and cities," in DATE, 2013, pp. 1149-1154.

[3] H.-l. Truong and S. Dustdar, "A survey on cloud-based sustainability governance systems," International Journal of Web Information Systems, vol. 8, no. 3, pp. 278 - 295, 2012.

[4] K.-Y. Wang, G. Lin, P. Chou, and A. Chou, "Leverage smart system services technology for smart green building management," in $E G G, 2012$.

[5] A. Schnieders and F. Puhlmann, "Variability mechanisms in e-business process families," in BIS, 2006.

[6] D. Patnaik, M. Marwah, R. Sharma, and N. Ramakrishnan, "Sustainable operation and management of data center chillers using temporal data mining," in KDD, 2009.

[7] L. Luskay, "Methods for Automated and Continuous Commissioning of Building Systems," Tech. Rep., 2003.

[8] T. Weng and Y. Agrawal, "From Buildings to Smart Buildings-Sensing and Actuation to Improve Energy Efficiency," IEEE Design \& Test of Computers, vol. 29, no. 4, pp. 36-44, 2012.

[9] G. P. Hancke, B. d. C. e. Silva, and G. P. Hancke, Jr., "The role of advanced sensing in smart cities," Sensors, 2012.

[10] A. Murguzur, G. Sagardui, K. Intxausti, and S. Trujillo, "Process variability through automated late selection of fragments," in CAiSE Workshops, 2013, pp. 371-385.

[11] M. Koning, C. Sun, M. Sinnema, and P. Avgeriou, "VxBPEL: supporting variability for Web services in BPEL," Information and Software Technology, vol. 51, no. 2, 2009.

[12] L. Baresi, S. Guinea, and L. Pasquale, "Service-Oriented Dynamic Software Product Lines," IEEE Computer, vol. 45, no. 10, pp. 42-48, 2012.

[13] S. Meerkamm, "Configuration of multi-perspectives variants," in BPM Workshops, 2010, pp. 277-288.

[14] M. L. Rosa, M. Dumas, A. H. M. ter Hofstede, and J. Mendling, "Configurable multi-perspective business process models," Information Systems, vol. 36, no. 2, pp. 313340, 2011. 